\title{
Position Stability For Phase Control Of The Preisach Hysteresis Model
}

\author{
Juan Manuel Cruz-Hernández*and Vincent Hayward ${ }^{\dagger}$
}

\begin{abstract}
Many systems with hysteresis are adequately represented by the Preisach model. Hysteresis in these systems can be very effectively reduced using the "phaser", an ideal frequency domain operator, in a feedback connection. The position stability of this type of control has not yet been established in spite of the experimental evidence that the resulting systems are stable. This paper shows the dissipativity for the relay operator, for the Preisach model, and then for the lead approximation to the phaser. We then give a proof of stability for the feedback connection of this phaser approximation with systems represented by the Preisach model of hysteresis.
\end{abstract}

\section{Introduction}

Actuators used in robotics and other applications often exhibit hysteresis which is detrimental to performance. For example, it is well known that most hydraulic actuators have significant hysteresis arising from the valve magnetic activation and friction in the seals [1]. Most strain-based actuators also have hysteresis, specifically actuators based on materials like piezoceramics, shape memory alloys, or magnetostrictive materials $[9,16]$.

To improve tracking precision, reduce steady-state error, and achieve other performance objectives, feedback controllers have been proposed (for recent contributions among others see [2, 3, 4, 17]), however little has been said about their stability. Gorbet et al. have shown the stability of velocity feedback control applied to the Preisach hysteresis model (Fig. 1a) [11], or in a similar configuration as in [10], but nothing was said about position control (Fig. 1b).

a

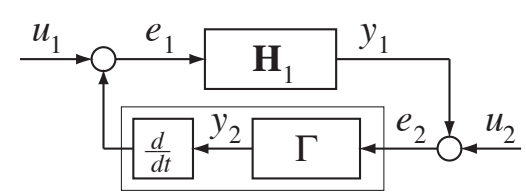

b

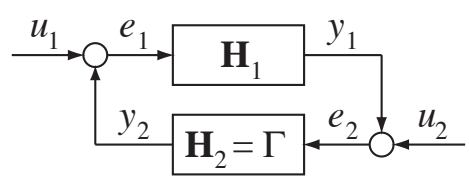

Figure 1: Control Systems Setup. a) Velocity configuration as in [11]. b) Position configuration as discussed here. $\mathbf{H}_{1}$ represents the controllers, $\mathbf{H}_{2}$ represents the plant and $\Gamma$ the Preisach model.

In this paper, we investigate the dissipativity of the Preisach model, in such a manner that stability of a feedback connection with a given controller can be tested using dissipativity theory [12]. The phaser is an ideal operator in the frequency domain which shifts the phase of a signal by a given amount but leaves the magnitude unchanged and was shown experimentally to be very effective at reducing hysteresis present in strain-based actuators [5]. An ideal phaser cannot be implemented

*Immersion Canada Inc., Montréal, Qc, H2X 2T7, Canada, mcruz@immersion.com.

${ }^{\dagger}$ Center for Intelligent Machines, McGill University, Montréal, Qc, H3A 2A7, Canada, hayward@cim.mcgill.ca. 
but can be approximated by a causal filter. One attractive possibility is a multiple lead network which is easy to design given performance specifications. Stability is investigated for this case, but other choices could be used as well as long as they satisfy the conditions stated in Section 3.

The Preisach model is a popular model for rate-independent hysteresis. It is constructed from the combination of an infinite number of elementary relays that serve as "atoms of hysteresis" [14]. All the relays receive the same input and there is one relay for each possible pair $(\alpha, \beta)$ of 'on' and 'off' switching thresholds. The outputs of all the relays are combined using a weighted sum over all the values of $\alpha$ and $\beta$. The weighting function entirely specifies a particular hysteresis. This model is briefly reviewed in the next section.

Dissipativity analysis begins with that of a single relay operator. This allows us to show the dissipativity of the Preisach model. We introduce a new representation of the relay operator, in terms of the feedback connection of two singular nonlinearities. With this, dissipativity can be obtained and extended to the dissipativity of the Preisach model. The lead approximation to the phaser can be shown to be dissipative too. In Section 4, we use a theorem by Hill and Moylan for the single-input single-output case to show that the feedback connection of the Preisach model and the approximation to the phaser is stable [12].

\section{The Preisach model}

The Preisach model results from the combination of relay operators such as the one shown in Fig. 2a.

a

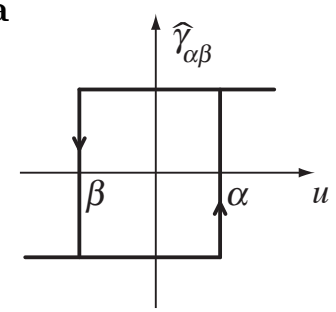

b

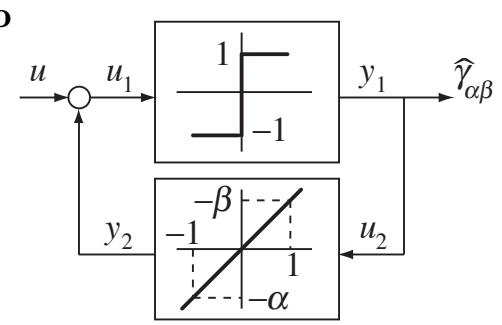

Figure 2: a) Relay operator. b) Equivalent representation of a relay using a feedback connection.

The real-valued weighting function $\mu_{\alpha, \beta}$, the Preisach function, with support $\mathbf{H}$ in $\alpha \geq \beta$, describes the relative contribution of an infinite collection of relays sharing the same input. The hysteresis output using the Preisach model is written:

$$
y(t)=\widehat{\Gamma}[u(t)]=\iint_{\mathbf{H}} \mu_{\alpha, \beta} \widehat{\gamma}_{\alpha \beta}[u(t)] \mathrm{d} \alpha \mathrm{d} \beta
$$

In $\alpha, \beta$ coordinates, each point of the half plane $\alpha \geq \beta$ is identified with only one particular $\widehat{\gamma}$ operator whose on and off switching values are respectively found at the $\alpha$ and $\beta$ coordinates of the point $\left(\widehat{\gamma}_{\alpha \beta}: \mathbb{R} \mapsto\{-1,1\}\right)$. The weighted output of a particular relay will be identified by $y_{\alpha \beta}(t)=\mu_{\alpha \beta} \widehat{\gamma}_{\alpha \beta}[u(t)]$. In practice, the support for $\mu_{\alpha \beta}$ is finite $[11,14]$. This means $\mu_{\alpha \beta}=0$ outside the triangle $\alpha=u_{2}, \beta=u_{1}, \alpha=\beta$. This can be thought of as restricting $\mathbf{H}$ to a triangle, as in Fig. 3b. 
Transactions of the CSME, Vol. 29, No. 2, pp. 129-142, 2005.

a

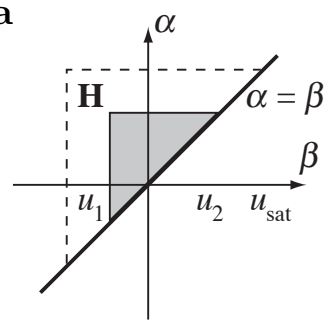

b

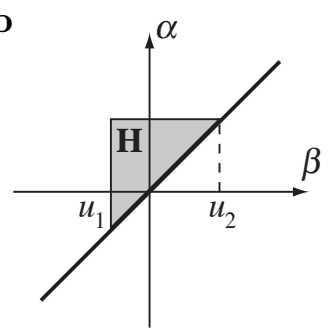

Figure 3: Limiting triangle for the Preisach model.

In systems which have nonzero slope outside the loop, such as some magnetic materials, degenerated relays must be added along the diagonal $\alpha=\beta$ in order to complete the model. Even in this case, physical limits such as control signal saturation, say at $u_{\text {sat }}$, allow $\mathbf{H}$ to be effectively bounded (Figure 3a). The gray area indicates the contributing relays, and the finite support $\mathbf{H}$ for $\mu$ is delimited by the dotted line.

For physical hysteresis (e.g. shape memory alloys, piezoceramics actuators, magnetostrictive actuators), it is reasonable to assume that $\mathbf{H}$ is bounded in $\alpha \geq \beta$. It is also clear that $\mu$ must be:

- non-negative, otherwise an increase in input could cause a decrease in output, and

- finite, since the major hysteresis loop is closed in the input-output plane.

Assumption 2.1 (Finite Support [11])

It will be assumed that the Preisach weighting function $\mu$ is finite, non-negative and has finite support: $\mathbf{H}=\left\{(\alpha, \beta) \mid \alpha \leq u_{2}, \beta \geq u_{1}, \alpha \geq \beta\right\}$, with $u_{2}>u_{1}$.

\subsection{Background}

Here, we follow the presentation of Subsection 2.2 in [11]. The mathematical background can be found for example in [8], however the notation used in [11] is employed for convenience.

$L_{2}(\mathbb{R})$ is the set of all functions $f: \mathbb{R} \mapsto \mathbb{R}$ such that $\int_{-\infty}^{\infty} f(t)^{2} \mathrm{~d} t<\infty$. It is known that $L_{2}(\mathbb{R})$ is a normed vector space with the $L_{2}$-norm defined by $\|f\|=\left(\int_{-\infty}^{\infty} f(t)^{2} \mathrm{~d} t\right)^{1 / 2}$. For any $T \geq 0$, consider the subset $L_{2}[0, T]$ of $L_{2}(\mathbb{R})$ defined by $L_{2}[0, T]=\{f: \mathbb{R} \mapsto \mathbb{R} \mid f(t)=0 \forall t \notin[0, T]\}$. The subset $L_{2}[0, T]$ with $\|f\|$ of $L_{2}(\mathbb{R})$ is also a normed vector space. For every $f \in L_{2}[0, T]$, $\|f\|=\left(\int_{-\infty}^{\infty} f(t)^{2} \mathrm{~d} t\right)^{1 / 2}=\left(\int_{0}^{T} f(t)^{2} \mathrm{~d} t\right)^{1 / 2}$. Consequently $\|f\|_{T}=\left(\int_{0}^{T} f(t)^{2} \mathrm{~d} t\right)^{1 / 2}$ is a norm for $L_{2}[0, T]$. Define the truncation of $f \in L_{2}(\mathbb{R})$, for any $T \geq 0$, to be $f_{T}(t)=f(t)$ for $0 \leq t \leq T$ and $f_{T}(t)=0$ for $t>T$. The extended space $L_{2 e}$ is composed of functions $f_{T}(t)$ for which $\int_{0}^{\infty} f_{T}(t)^{2} \mathrm{~d} t<\infty$. Given two functions $f$ and $g$ in $L_{2}(\mathbb{R})$, the inner product of these two functions is defined as $\langle f, g\rangle=\int_{-\infty}^{\infty} f(t) g(t) \mathrm{d} t$. For $f$ and $g$ in $L_{2}[0, T],\langle f, g\rangle_{T}=\left\langle x_{T}, y_{T}\right\rangle=\int_{-\infty}^{\infty} f(t) g(t) \mathrm{d} t=$ $\int_{0}^{T} f(t) g(t) \mathrm{d} t$.

Hysteresis is a nonlinearity with memory which implies that the output may be affected by the entire history of past inputs. The same input applied to a hysteretic system at $t_{0}>0$ may yield different outputs, depending on the input history over $\left[0, t_{0}\right]$. This makes it difficult to define a "relaxed state", as is typically done in passivity analysis. This is because the natural physical interpretation of relaxation: setting the input to zero and letting $t \rightarrow \infty$, may lead to different equilibria at any point along the axis where the input is zero. The relaxed state for such a system 
is not unique, and may correspond to an entire set of outputs. Relations are introduced in order to deal with the multi-valued nature of the output, and to avoid having to define a unique relaxed state.

Definition 2.1 (Relation [11])

$A$ relation $H$ on $L_{2}[0, T]$ defines a set of ordered pairs $(x, y) \in L_{2}[0, T] \times L_{2}[0, T]$. The domain and range of $H$ can be defined as follows:

$$
\begin{aligned}
& D o(H)=\left\{x \in L_{2}[0, T] \mid \exists y \in L_{2}[0, T] \text { s.t. } y=H x\right\}, \\
& R a(H)=\left\{y \in L_{2}[0, T] \mid \exists x \in L_{2}[0, T] \text { s.t. } y=H x\right\}
\end{aligned}
$$

A relation may be multi-valued, meaning that for any $x \in D o(H)$, there may be several $y \in$ $L_{2}[0, T]$ such that $y=H x$, and this multi-valued property makes the relations useful in describing hysteretic systems. When something is said to hold (or is required to hold) for $H x$, without qualification, it will be understood that it holds (or is required to hold) for all possible outputs corresponding to the input $x$. It is also important to note that $H$ need not be defined on the whole of $L_{2}[0, T]$.

\section{Definition 2.2 (Sobolev Space)}

The Sobolev Space $W_{1}^{2}[0, T]$ is the space of functions $u \in L_{2}[0, T]$ for which the Sobolev norm is finite:

$$
\|u\|_{W_{1}^{2}}=\sqrt{\int_{0}^{T}\left(\dot{u}^{2}+u^{2}\right) \mathrm{d} t} .
$$

Clearly, if $u \in W_{1}^{2}[0, T]$, then $\dot{u} \in L_{2}[0, T]$.

The following definitions are needed for the next theorem [11]. Define $R_{\alpha}$ and $R_{\beta}$, horizontal and vertical strips in $\mathbf{H}$, and the function $k$ as follows:

$$
\begin{aligned}
R_{\alpha}(\lambda, \xi) & \triangleq\{(\alpha, \beta) \in \mathbf{H} \mid \lambda \leq \alpha \leq \lambda+\xi\} \\
R_{\beta}(\lambda, \xi) & \triangleq\{(\alpha, \beta) \in \mathbf{H} \mid \lambda \leq \beta \leq \lambda+\xi\} \\
k(\xi) & \triangleq \sup _{\lambda \in \mathbf{R}, R \in\left\{R_{\alpha}, R_{\beta}\right\}} \iint_{R} \mu_{\alpha \beta} \mathrm{d} \alpha \mathrm{d} \beta
\end{aligned}
$$

\section{Theorem 2.1 (in [11])}

Suppose $\mu$ is finite and non-negative on $\mathbf{H}$. The Preisach operator maps $\mathbf{W}_{1}^{2}[0, T]$ into itself if and only if there exists $C>0$ such that $k(\xi) \leq C \xi$ for all $\xi>0$.

The conditions of the above theorem hold if the support $\mathbf{H}$ is finite, and $\mu$ is finite and non-negative.

\section{Corollary 2.1 (Claim 2.1 in [11])}

If $\mathbf{H}$ is finite and $0 \leq \mu_{\alpha \beta}<\infty$ for all $(\alpha, \beta) \in \mathbf{H}$, then there exists $C>0$ such that $k(\xi) \leq C \xi$ for all $\xi>0$.

Given the above Claim and Assumption 2.1, Theorem 2.1 applies and $\widehat{\Gamma}: \mathbf{W}_{1}^{2}[0, T] \mapsto \mathbf{W}_{1}^{2}[0, T]$. 


\section{Transactions of the CSME, Vol. 29, No. 2, pp. 129-142, 2005.}

\subsection{The Phaser Controller [5]}

Assume that the input to a hysteretic system varies periodically between two values. The Preisach model predicts that a loop is produced in the input-output graph. It is then possible to speak of phase shift between input and output. The loop and the output will have the same period as the input and the effect can be viewed as phase lag between input and output. Moreover, the phase lag angle $\phi$ is constant if rate independence holds [6]. Consider the following:

\section{Definition 2.3 (in [5])}

$A$ Phaser $\mathbf{L}_{\mathrm{pa}}$ is an operator that shifts a periodic input signal by a constant angle, and has unity gain.

The phaser operator can be seen as the counterpart of the proportional controller for which the phase is zero but the magnitude is adjusted according to design specifications. The phaser keeps the magnitude constant but applies a given phase shift at all frequencies according to design specifications.

The effect of the phaser used in a feedback loop can be intuitively appreciated by considering a Fourier series expansion of the input signal. Periodic signals can be decomposed into a combination of sinusoidal signals with frequencies $\frac{1}{T}, \frac{2}{T}, \ldots, \frac{k}{T}$ where $\frac{1}{T}$ is the fundamental frequency. All components of a signal distorted by rate independent hysteresis are shifted the same way and all are corrected the same way. Since it is only an approximation, the correction cannot be perfect, so an error term $e$ (distortion) is formed for further correction by feedback. Moreover, when dealing with nonlinear systems, in some cases, it is possible to only consider the fundamental component of the output (as in the describing function analysis).

A phaser is an idealization that cannot be implemented because no causal physical system can provide a constant phase shift over an infinite frequency range. However, a phaser can be approximated by causal linear filters. Among other choices, the following transfer function represents a phaser controller approximated by a lead network, with all the factors covering adjacent frequency bands:

$$
\widehat{\mathbf{L}}_{\mathrm{pa}}(s)=\left(\frac{s+p_{n}}{s+q_{n}}\right)\left(\frac{s+p_{n-1}}{s+q_{n-1}}\right) \ldots\left(\frac{s+p_{1}}{s+q_{1}}\right),
$$

where the $p_{i}$ and $q_{i}$ are greater than zero for $i=1, \ldots, n$. This fraction can be rearranged such that $p_{i}>q_{i}$ for all $i$ 's. Properly designed, the phase of $\widehat{\mathbf{L}}_{\mathrm{pa}}(s)$ will be nearly constant within a wide frequency range. A phaser $\widehat{\mathbf{L}}_{\mathrm{pa}}(j \omega)$ in the complex plane has a Nyquist plot as in Fig. 4 for any $n$.

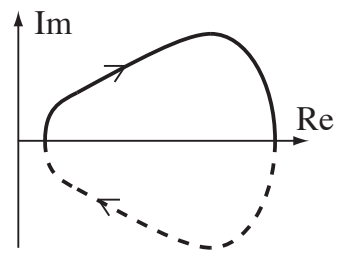

Figure 4: Nyquist plot of the linear approximation to the phaser as in Eqn (3).

It is clear that the real part of $\widehat{\mathbf{L}}_{\mathrm{pa}}(j \omega)$ is always positive and different from zero for all $\omega$, and that it has a well defined lower bound (it is continuously bounded). Also note that $\left\|\widehat{\mathbf{L}}_{p a}(j \omega)\right\|$ is 
bounded above by 1 for all $\omega$. The product $\widehat{\mathbf{L}}_{\mathrm{pa}}(j \omega) \widehat{\mathbf{L}}_{\mathrm{pa}}(-j \omega)$ is also positive since each factor in Eqn (3) will be multiplied by its corresponding complex conjugate, yielding a positive number.

$$
\widehat{\mathbf{L}}_{\mathrm{pa}}(j w) \widehat{\mathbf{L}}_{\mathrm{pa}}(-j w)=\left(\frac{j \omega+p_{n}}{j \omega+q_{n}}\right)\left(\frac{-j \omega+p_{n}}{-j \omega+q_{n}}\right) \ldots\left(\frac{j \omega+p_{1}}{j \omega+q_{1}}\right)\left(\frac{-j \omega+p_{1}}{-j \omega+q_{1}}\right) .
$$

Therefore the product will be also bounded by a finite positive number and will be different from zero. Thus we have

$$
\forall \omega, \quad A=2 \max _{\omega}\left(\frac{\widehat{\mathbf{L}}_{\mathrm{pa}}(j \omega) \widehat{\mathbf{L}}_{\mathrm{pa}}(-j \omega)}{\operatorname{Re}\left[\widehat{\mathbf{L}}_{\mathrm{pa}}(j \omega)\right]}\right)>0, \quad B=\max _{\omega}\left(\frac{\pi}{\operatorname{Re}\left[\widehat{\mathbf{L}}_{\mathrm{pa}}(j \omega)\right]}\right)>0
$$

which we will use in Section 3.3.

\section{Dissipativity}

Definition 3.1 (Dissipativity, modified from [13])

Given a relation $G$ on $L_{2 e}$, with $y=G u$, and $q, r, s \in \mathbb{R}, G$ is said to be $(q, r, s)$-dissipative iff,

$$
\langle y, q y\rangle_{T}+\langle y, s u\rangle_{T}+\langle u, r u\rangle_{T} \geq 0
$$

for all $u \in D o(G)$ and all $T \geq 0$.

\subsection{Dissipativity of the Relay Operator}

Consider one single relay $y=y_{\alpha \beta}$ and the scalars $q, s$, and $r$. Assume $u$ is in the Sobolev space, i.e. $u \in \mathbf{W}_{1}^{2}[0, T]$, and the Preisach function $\mu_{\alpha \beta}$ is finite, then $y=y_{\alpha \beta}$ is also finite. A relay operator can be replaced by a feedback connection as in Fig. $2 \mathrm{~b}$. If $m=\frac{\alpha-\beta}{2}$ and $b=-\frac{\alpha+\beta}{2}$ then, this system is equivalent to that in Fig. 2a. In both cases $y_{2}(t)=\frac{\alpha-\beta}{2} \operatorname{sgn}\left(u_{1}(t)\right)-\frac{\alpha+\beta}{2}$. Also, in Fig. 2b, the following holds:

$$
\begin{aligned}
u(t) & =u_{1}(t)-y_{2}(t) \\
u_{1}(t) u_{2}(t) & =\left|u_{1}(t)\right| \\
y_{2}(t) & =m u_{2}(t)+b .
\end{aligned}
$$

Note also that a weighted relay operator is represented by $y_{\alpha \beta}=\mu_{\alpha \beta} \hat{\gamma}_{\alpha \beta}$. For simplicity take $\mu_{\alpha \beta}=1$ and $y=\hat{\gamma}_{\alpha \beta}$, then the product $u y$ is:

$$
\begin{aligned}
u(t) y(t) & =u(t) \hat{\gamma}_{\alpha \beta}=u_{1}(t) y_{1}(t)-u_{2}(t) y_{2}(t) \\
& =u_{1}(t) \operatorname{sgn}\left(u_{1}(t)\right)-m u_{2}^{2}(t)-b u_{2}(t) \\
& =\left|u_{1}(t)\right|-m u_{2}^{2}(t)-b u_{2}(t) .
\end{aligned}
$$

Expressing $-m u_{2}^{2}(t)-b u_{2}(t)$ in terms of $\alpha$ and $\beta$, with $u_{2}^{2}(t)=1, u_{2}(t)= \pm 1, m=\frac{\alpha-\beta}{2}$, and $b=-\frac{\alpha+\beta}{2}$, we get

$$
\begin{aligned}
-m u_{2}^{2}(t)-b u_{2}(t) & =-m-b u_{2}(t) \\
& =\left\{\begin{array}{l}
-m-b=-\frac{\alpha-\beta}{2}+\frac{\alpha+\beta}{2}=\beta \\
-m+b=-\frac{\alpha-\beta}{2}-\frac{\alpha+\beta}{2}=-\alpha \quad \text { for } \quad u_{2}=1
\end{array}\right.
\end{aligned}
$$


Therefore Eqn (10) can be expressed as,

$$
u(t) y(t)=\left\{\begin{array}{lll}
\left|u_{1}(t)\right|+\beta & \text { for } & u_{2}=1 \\
\left|u_{1}(t)\right|-\alpha & \text { for } & u_{2}=-1
\end{array}\right.
$$

Now, the dissipativity result.

\section{Lemma 3.1 (Dissipativity of a relay operator)}

Let the real-valued function $u(t)$, and the relation $\gamma_{\alpha \beta},\left(y(t)=\gamma_{\alpha \beta}[u(t)]\right)$ be in $\mathbf{W}_{1}^{2}[0, T]$. The weighted relay operator is dissipative with respect to:

$$
\begin{aligned}
q & =0, \\
\text { for some } r & \gg 1, \\
\text { and } s & =\min _{\left(\alpha, \beta \in \mathbf{H}, \forall \mu_{\alpha \beta}\right)} \frac{1}{\max \left(\mu_{\alpha \beta}|\alpha|, \mu_{\alpha \beta}|\beta|\right)}>0 .
\end{aligned}
$$

That is:

$$
s \int_{0}^{T} u(t) y(t) d t+r \int_{0}^{T} u^{2}(t) \mathrm{d} t \geq 0
$$

$\forall t, 0 \leq t \leq T, \forall T \geq 0$, and $u \in D o\left(\gamma_{\alpha \beta}\right)$.

Proof: For all $T \geq 0$, and all $\alpha$ and $\beta$ in $\mathbf{H}$, and $u \in D o(H)$, the following equality holds,

$$
r \int_{0}^{T} u^{2}(t) d t+s \int_{0}^{T} u(t) y(t) \mathrm{d} t=\int_{0}^{T}\left(r u_{2}^{2}(t)+s\left|u_{1}(t)\right|-s m u_{2}^{2}(t)-s b u_{2}(t)\right) \mathrm{d} t,
$$

by using Eqn (10). We must prove that the right hand side of Eqn (17) is greater than or equal to zero. Using Eqn (12), it is equivalent to show that

$$
r u_{2}^{2}(t)+s\left|u_{1}(t)\right|-s m u_{2}^{2}(t)-s b u_{2}(t)=\left\{\begin{array}{lll}
r u^{2}(t)+s\left|u_{1}(t)\right|+s \beta & \text { for } u_{2}(t)=1, \\
r u^{2}(t)+s\left|u_{1}(t)\right|-s \alpha & \text { for } & u_{2}(t)=-1 .
\end{array}\right.
$$

Consider now both cases independently, choose $r>0$ and $s>0$, and take into account that $u_{1}(t)=u(t)+y_{2}(t)$,

$$
u_{2}(t)=1
$$

For this case $u_{1}(t)=u(t)-\beta$, then we have

$$
r u^{2}(t)+s\left|u_{1}(t)\right|+s \beta=r u^{2}(t)+s|u(t)-\beta|+s \beta .
$$

Consider here the following subcases:

- $\beta \geq 0$ : In this case $r u^{2}(t)+s|u(t)-\beta|+s \beta \geq 0$ for any value of $u(t)$.

- $\beta<0$ : We will make the value of $s$ s.t. $s|\beta|<1$ for all $\beta$, and the value of $r \gg 1$. Then if 
- $u(t) \geq 0$ : Eqn (18) can be expressed as

$$
r u^{2}(t)+s|u(t)+| \beta||-s|\beta| \geq 0
$$

$-u(t) \leq-1$ : Since $r \gg 1$ and $s|\beta|<1$, Eqn (18) is greater than zero.

$--1<u(t)<0$ : Eqn $(18)$ attains its minimum for $u(t) \in(-1,0)$ and any $\beta$. Because $r \gg 1$ and $s|\beta|<1$, Eqn (18) is always positive.

$u_{2}(t)=-1$

For this case $u_{1}(t)=u(t)-\alpha$, then we have

$$
r u^{2}(t)+s\left|u_{1}(t)\right|-s \alpha=r u^{2}(t)+s|u(t)-\alpha|-s \alpha .
$$

Consider the following subcases and use similar arguments as for $u_{2}(t)=1$

- $\alpha \leq 0: r u^{2}(t)+s|u(t)-\alpha|-s \alpha \geq 0$, for any $u(t)$

- $\alpha>0$ : Again take $s$ such that $s|\alpha|<1$ for all $\alpha$, and $r \gg 1$, then

- $u(t) \leq 0$ : Eqn $(20)$ is equal to $\left(r u^{2}(t)+s|u(t)-\alpha|-s \alpha\right) \geq 0$

- $u(t) \geq 1$ and $u(t) \in(-1,0)$ : Similarly, Eqn $(20)$ attains its minimum for $u(t) \in(0,1)$ and any $\alpha$. Because $r \gg 1$ and $s|\alpha|<1$, Eqn (20) is always positive.

The value of $s$ can be:

$$
s=\min _{(\alpha, \beta \in \mathbf{H})} \frac{1}{\max (|\alpha|,|\beta|)}
$$

which clearly satisfies the conditions stated above, and it is never zero since $\alpha$ and $\beta$ are finite numbers. It has been proven that the argument of the integral in the r.h.s. of Eqn (17) is greater than or equal to zero for all $\alpha$ and $\beta$ in $\mathbf{H}$ and $u \in D o(\mathbf{H})$. Therefore Eqn (17) is always positive:

$$
r \int_{0}^{T} u^{2}(t) \mathrm{d} t+s \int_{0}^{T} u(t) y(t) \mathrm{d} t=\int_{0}^{T}\left(r u_{2}^{2}(t)+s\left|u_{1}(t)\right|-s m u_{2}^{2}(t)-s b u_{2}(t)\right) \mathrm{d} t \geq 0
$$

for any $T \geq 0$.

For a weighted relay operator the result holds, since $y$ is multiplied by an arbitrary $\mu_{\alpha \beta}$ which is always positive. This will alter the expression in the r.h.s. of Eqn (17) where $s$ will be multiplied by a positive and finite number $\mu_{\alpha \beta}$. We can take $s \mu_{\alpha \beta}|\alpha|<1$ and $s \mu_{\alpha \beta}|\beta|<1$ for all possible values of $\alpha$ and $\beta$. Now $s$ can be expressed as:

$$
s=\min _{\left(\alpha, \beta \in \mathbf{H}, \forall \mu_{\alpha \beta}\right)} \frac{1}{\max \left(\mu_{\alpha \beta}|\alpha|, \mu_{\alpha \beta}|\beta|\right)}
$$

This concludes the proof. 


\subsection{Dissipativity of the Preisach Model}

The dissipativity of the Preisach model is now derived.

\section{Lemma 3.2 (Dissipativity of the Preisach model)}

Let the real-valued function $u(t)$, and the relation $\Gamma,(y(t)=\Gamma[u(t)])$ be in $\mathbf{W}_{1}^{2}[0, T]$. The Preisach model is dissipative with respect to $q=0, r=s_{\text {relay }} r_{\text {relay }} \iint_{\mathbf{H}} \mu_{\alpha \beta} d \alpha d \beta>0$ and $s=s_{\text {relay }}>0$, i.e.

$$
s \int_{0}^{T} u(t) y(t) d t+r \int_{0}^{T} u^{2}(t) \mathrm{d} t \geq 0
$$

for all $0 \leq t \leq T$, for all $T \geq 0$ and $u \in D o(\Gamma)$ and all points in $\mathbf{H}$. $s_{\text {relay }}$ and $r_{\text {relay }}$ are the values found in lemma 3.1.

Proof: First evaluate:

$$
\begin{aligned}
s \int_{0}^{T} u(t) y(t) \mathrm{d} t & =s \int_{0}^{T} u(t)\left(\iint_{\mathbf{H}} y_{\alpha \beta} d \alpha d \beta\right) \mathrm{d} t \\
& =s \int_{0}^{T} u(t)\left(\iint_{\mathbf{H}} \mu_{\alpha \beta} \hat{\gamma}_{\alpha \beta} d \alpha d \beta\right) \mathrm{d} t \\
& =s \iint_{\mathbf{H}} \mu_{\alpha \beta}\left(\int_{0}^{T} u(t) \hat{\gamma}_{\alpha \beta} \mathrm{d} t\right) \mathrm{d} \alpha \mathrm{d} \beta
\end{aligned}
$$

Using the fact that a relay operator is dissipative for every point in $\mathbf{H}$, and all $u \in D o(\Gamma)$ as in lemma 3.1, yields

$$
\begin{aligned}
s_{\text {relay }} \int_{0}^{T} u(t) y(t) d t & \geq s_{\text {relay }} \iint_{\mathbf{H}} \mu_{\alpha \beta}\left(-r_{\text {relay }} \int_{0}^{T} u^{2}(t) \mathrm{d} t\right) \mathrm{d} \alpha \mathrm{d} \beta \\
& =-\int_{0}^{T} u^{2}(t) d t \cdot s_{\text {relay }} \iint_{\mathbf{H}} \mu_{\alpha \beta} r_{\text {relay }} \mathrm{d} \alpha \mathrm{d} \beta \\
& \geq-r \int_{0}^{T} u^{2}(t) \mathrm{d} t
\end{aligned}
$$

The value $r=s_{\text {relay }} r_{\text {relay }} \iint_{\mathbf{H}} \mu_{\alpha \beta} \mathrm{d} \alpha \mathrm{d} \beta$ is always positive since $\mu_{\alpha \beta}$ is positive and finite. Therefore the Preisach model is dissipative.

\subsection{Dissipativity Of The Lead Approximation Of A Phaser}

To prove that the lead approximation to the phaser is dissipative, a proof using Parseval's theorem similar to the proofs of passivity in linear systems is used $[8,18]$. We consider that the transfer function that approximates the ideal phaser is rational with relative degree 0 , and causal. This compensates a hysteretic system by providing phase in the feedback that is always smaller then or equal to $90^{\circ}$.

Lemma 3.3 (Modified from [8])

The linear system $\mathbf{L}: \mathbf{W}_{1}^{2} \rightarrow \mathbf{W}_{1}^{2}$ defined by

$$
\mathbf{L} u=l * u, \quad \text { where } \quad \mathbf{L} \quad \text { is causal and } u \in \mathbf{W}_{1}^{2}
$$

is said to be passive iff $\operatorname{Re}[\mathbf{L}(j \omega)] \geq 0, \forall \omega \in \mathbf{R}$. 


\section{Transactions of the CSME, Vol. 29, No. 2, pp. 129-142, 2005.}

The lead approximation to the phaser is causal and passive, i.e. strictly stable. We use this result to show that the controller is also dissipative.

\section{Lemma 3.4 (Dissipativity of the lead approximation)}

The lead approximation to the phaser $\widehat{\mathbf{L}}_{\mathrm{pa}}: \mathbf{W}_{1}^{2} \rightarrow \mathbf{W}_{1}^{2}$, causal and strictly stable (passive), is dissipative with respect to

$$
\begin{aligned}
& r<0 \\
& q \leq-\alpha r_{2}<0 \quad \text { and } \\
& s \geq-q A-r B, \quad A=2 \max _{\omega}\left(\frac{\widehat{\mathbf{L}}_{\mathrm{pa}}(j \omega) \widehat{\mathbf{L}}_{\mathrm{pa}}(-j \omega)}{\operatorname{Re}\left[\widehat{\mathbf{L}}_{p a}(j \omega)\right]}\right), \quad B=\max _{\omega}\left(\frac{\pi}{\operatorname{Re}\left[\widehat{\mathbf{L}}_{p a}(j \omega)\right]}\right), \\
& \text { i.e. } \quad r \int_{0}^{T} u(t)^{2} d t+s \int_{0}^{T} y(t) u(t) d t+q \int_{0}^{T} y(t)^{2} \mathrm{~d} t \geq 0
\end{aligned}
$$

$\forall t, 0 \leq t \leq T, \forall T \geq 0$ and $u \in \mathbf{W}_{1}^{2}, r_{2}$ being the $r$ found in the dissipativity of the Preisach model.

The value of $q$ is taken to meet the stability conditions of the feedback connection of the Preisach model with a dissipative controller as will be shown in the next section. The value of $s$ and $r$ are obtained as follows.

Assume that $y(j \omega) / u(j \omega)=\widehat{\mathbf{L}}_{\mathrm{pa}}(j \omega)$, is initially at rest $(y \equiv 0)$. If the input $u$ is zero for all $t>T$ and $t<0$, then using the passivity property of the system we have:

$$
\begin{aligned}
\int_{0}^{T} y(t) u(t) \mathrm{d} t & =\int_{-\infty}^{\infty} y(t) u(t) \mathrm{d} t \\
& =\frac{1}{2 \pi} \int_{-\infty}^{\infty} y(j \omega) u^{*}(j \omega) d \omega .
\end{aligned}
$$

The first equality holds because $u(t)=0$ for $t>T$ and for $t<0$. The second equality comes from the Parseval's theorem, with the superscript * referring to complex conjugation. Since $y(j \omega)=$ $\widehat{\mathbf{L}}_{\mathrm{pa}}(j \omega) u(j \omega)$, then

$$
\int_{0}^{T} y(t) u(t) \mathrm{d} t=\frac{1}{2 \pi} \int_{-\infty}^{\infty} \widehat{\mathbf{L}}_{\mathrm{pa}}(j \omega)|u(j \omega)|^{2} \mathrm{~d} \omega
$$

Since $\mathbf{L}_{\mathrm{pa}}$ is the transfer function of a real system, its coefficients are real and thus $\widehat{\mathbf{L}}_{\mathrm{pa}}(-j \omega)=$ $\left[\widehat{\mathbf{L}}_{\mathrm{pa}}(j \omega)\right]^{*}$, then

$$
\int_{0}^{T} y(t) u(t) d t=\frac{1}{\pi} \int_{0}^{\infty} \operatorname{Re}\left[\mathbf{L}_{\mathrm{pa}}(j \omega)\right]|u(j \omega)|^{2} \mathrm{~d} \omega
$$

(This also follows from the fact that the system is passive).

Note also that

$$
\begin{aligned}
\int_{0}^{T} y(t)^{2} d t & =\frac{2}{\pi} \int_{0}^{\infty} \widehat{\mathbf{L}}_{\mathrm{pa}}^{*}(j \omega) u^{*}(j \omega) \widehat{\mathbf{L}}_{\mathrm{pa}}(j \omega) u(j \omega) \mathrm{d} \omega \\
& =\frac{2}{\pi} \int_{0}^{\infty} \widehat{\mathbf{L}}_{\mathrm{pa}}(j \omega) \widehat{\mathbf{L}}_{\mathrm{pa}}(-j \omega)|u(j \omega)|^{2} \mathrm{~d} \omega
\end{aligned}
$$




\section{Transactions of the CSME, Vol. 29, No. 2, pp. 129-142, 2005.}

which is obtained after using the same arguments $(u(t)=0$ for $t>T$ and $t<0$ and the Parseval's theorem).

Expressions (30) and (32) are used to prove the dissipativity of the lead approximation to the phaser. It has to be shown that,

$$
\begin{aligned}
r & \int_{0}^{T} u(t)^{2} d t+s \int_{0}^{T} y(t) u(t) d t-q \int_{0}^{T} y(t)^{2} \mathrm{~d} t \\
& =r \int_{-\infty}^{\infty}|u(j \omega)|^{2} d \omega+s \frac{1}{\pi} \int_{-\infty}^{\infty} \operatorname{Re}\left[\widehat{\mathbf{L}}_{\mathrm{pa}}(j \omega)\right]|u(j \omega)|^{2} d \omega+q \frac{2}{\pi} \int_{-\infty}^{\infty} \widehat{\mathbf{L}}_{\mathrm{pa}}(j \omega) \widehat{\mathbf{L}}_{\mathrm{pa}}(-j \omega)|u(j \omega)|^{2} \mathrm{~d} \omega \\
& =\int_{-\infty}^{\infty}|u(j \omega)|^{2}\left[s \frac{1}{\pi} \operatorname{Re}\left[\widehat{\mathbf{L}}_{\mathrm{pa}}(j \omega)\right]+q \frac{2}{\pi} \widehat{\mathbf{L}}_{\mathrm{pa}}(j \omega) \widehat{\mathbf{L}}_{\mathrm{pa}}(-j \omega)+r\right] \mathrm{d} \omega \geq 0
\end{aligned}
$$

The previous inequality will be greater than or equal to zero if $r<0$ and

$$
s \geq \frac{-2 q \widehat{\mathbf{L}}_{p a}(j \omega) \widehat{\mathbf{L}}_{p a}(-j \omega)-r \pi}{\operatorname{Re}\left[\widehat{\mathbf{L}}_{\mathrm{pa}}(j \omega)\right]}
$$

for all $\omega$. Note that $\operatorname{Re}\left[\widehat{\mathbf{L}}_{\mathrm{pa}}(j \omega)\right]>0$ for all $\omega$, and $0<\widehat{\mathbf{L}}_{\mathrm{pa}}(j \omega) \widehat{\mathbf{L}}_{\mathrm{pa}}(-j \omega)<\infty$. Therefore, $s$ can be expressed as

$$
s \geq-q A-r B, \quad A=2 \max _{\omega}\left(\frac{\widehat{\mathbf{L}}_{\mathrm{pa}}(j \omega) \widehat{\mathbf{L}}_{\mathrm{pa}}(-j \omega)}{\operatorname{Re}\left[\widehat{\mathbf{L}}_{\mathrm{pa}}(j \omega)\right]}\right), \quad B=\max _{\omega}\left(\frac{\pi}{\operatorname{Re}\left[\widehat{\mathbf{L}}_{\mathrm{pa}}(j \omega)\right]}\right) .
$$

Given Eqn (5), Eqn (34) is a sufficient condition for passivity and for dissipativity.

\section{Stability}

Consider Fig. 5 to be the configuration of the feedback system.

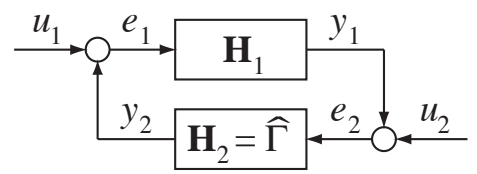

Figure 5: Feedback configuration.

\section{Theorem 4.1 (in [12])}

Suppose that the two subsystems $\mathbf{H}_{1}$ and $\mathbf{H}_{2}=\widehat{\Gamma}$ are dissipative with respect to supply rates

$$
w_{i}\left(u_{i}, y_{i}\right)=y_{i}^{\prime} q_{i} y_{i}+2 y_{i}^{\prime} s_{i} u_{i}+u_{i}^{\prime} r_{i} u_{i}, \quad i=1,2
$$

(consider $\mathrm{H}_{1}$ and $\mathrm{H}_{2}=$ to be relations). Then the feedback system is stable (asymptotically stable) if the matrix

$$
\hat{Q}=\left[\begin{array}{cc}
q_{1}+\alpha r_{2} & -s_{1}+\alpha s_{2} \\
-s_{1}+\alpha s_{2} & r_{1}+\alpha q_{2}
\end{array}\right]
$$

is negative semidefinite (negative definite) for some $\alpha>0$. 
The dissipativity conditions of the previous theorem when $\mathbf{H}_{2}$ is the Preisach model and $\mathbf{H}_{1}$ is the lead approximation to the phaser are satisfied given the conditions of Section 3. The following Subsection presents the proof of the stability of the feedback connection of these two subsystems.

\subsection{Stability with the Lead Approximation to a Phaser}

It was found in the previous section that both the Preisach model and the lead approximation to the phaser are dissipative. It remains to be shown that the matrix $\widehat{Q}$ is negative semidefinite (negative definite) for some $\alpha>0$. Also note that since $\mathbf{H}_{1}$ and $\mathbf{H}_{2}$ are relations, the input $u_{2}$ in Figure 5 , will be in $\mathbf{W}_{1}^{2}[0, T]$ and it will also offset the initial conditions on $y_{1}$, so that $e_{2} \in D o\left(\mathbf{H}_{2}\right)$ and the dissipativity of $\mathbf{H}_{2}$ can be exploited.

Let $\mathbf{H}_{1}=\widehat{\mathbf{L}}_{\mathrm{pa}}$ and $\mathbf{H}_{2}$ be the Preisach model. From Section 3, it is known that the Preisach model is dissipative with respect to $q_{2}=0, r_{2}>0$ and $s_{2}=s_{\text {relay }}>0$. For the lead approximation, $s_{1} \geq-q_{1} A-r_{1} B$ as in (34), $q_{1} \leq-\alpha r_{2}$, and $r_{1}<0$.

Theorem 4.1 can be easily applied if the terms $\widehat{Q}_{12}$ and $\widehat{Q}_{21}$ of the matrix $\widehat{Q}$ are equal to zero. This is achieved for an $\alpha>0$, if $s_{1}-\alpha s_{2}=0$ as follows:

$$
\begin{aligned}
\alpha & =\frac{s_{1}}{s_{2}} \geq \frac{-q_{1} A-r_{1} B}{s_{2}} \\
& \geq \frac{\alpha r_{2} A-r_{1} B}{s_{2}} \quad \text { since } \quad-q_{1} \geq \alpha r_{2} \\
\alpha & \geq \frac{-r_{1} B}{s_{2}-r_{2} A},
\end{aligned}
$$

and since $-r_{1}>0$, we just need to set the value of $s_{2}$ to be $s_{2}>\max \left\{s_{\text {relay }}, r_{2} A\right\}$, to ensure that $\alpha$ will be always positive. Then the matrix $\hat{Q}$ can assume the value

$$
\widehat{Q}=\left[\begin{array}{cc}
q_{1}+\alpha r_{2} & 0 \\
0 & r_{1}
\end{array}\right]
$$

Since $r_{1}<0$, we see that when $q_{1}=-\alpha r_{2}$, the matrix is negative semidefinite (eigenvalues smaller than or equal to zero), and when $q_{1}<-\alpha r_{2}$ it is negative definite (eigenvalues smaller than zero). Hence the feedback connection of the lead approximation to the phaser with the Preisach model is stable (asymptotically stable).

\section{Conclusion}

There is strong experimental evidence that the feedback connection of a system with hysteresis and a lead-type compensator is stable. The lead approximation to the phaser has been used successfully in the reduction of hysteresis in a piezoceramic actuator [6], and a shape memory alloy actuator [7]. Different types of lead controllers were shown to greatly improve the control of a system with magnetic hysteresis as reported in [15]. In all these cases, the feedback connection was stable.

Given this evidence, it was felt that it was possible to prove the stability of the Preisach model with a certain types of dissipative controllers, and in particular for the lead approximation of a phaser controller. The results obtained for the feedback-equivalent relay operators are of particular interest and further research should be focused on the stability of the Preisach model with more general classes of feedback controllers. 


\section{Acknowledgements}

The late Prof. G. Zames suggested replacing the relay operator by simpler feedback connections.

This research was partly funded by an operating grant from the Natural Sciences and Engineering Council of Canada and by the Institute for Robotics and Intelligent Systems part of Canada's Centres of Excellence Program (NCE).

\section{References}

[1] B. Boulet, L. K. Daneshmend, V. Hayward, and C. Nemri. System identification and modelling of a high performance hydraulic actuator. In R. Chatila and G. Hirzinger, editors, Experimental Robotics 2, Lecture Notes in Control and Information Sciences. Springer Verlag, 1993.

[2] B. M. Chen, T. H. Lee, C. C. Hang, Y. Guo, and S. Weerasooriya. An $\mathrm{H}^{\infty}$ almost disturbance decoupling robust controller design for a piezoelectric bimorph actuator with hysteresis. IEEE T. On Control Systems Technology, 7(7):160-194, 1999.

[3] G. S Choi, Y. A. Lim, and G. H. Choi. Tracking position control of piezoelectric actuators for periodic reference inputs. Mechatronics, 12:669-684, 2002.

[4] M. L. Corradini and G. Parlangeli. Robust stabilization of nonlinear uncertain plants with hysteresis in the actuator: A sliding mode approach. In IEEE Int. Conf. on Systems, Man and Cybernetics, volume 3, pages 500-505, 2002.

[5] J. M. Cruz-Hernandez and V. Hayward. Phase control approach to hysteresis reduction. IEEE T. On Control Systems Technology, 9(1):17-26, 2001.

[6] J.M. Cruz-Hernández and V. Hayward. On the linear compensation of hysteresis. In 36th IEEE Conference on Decision and Control, volume 1, pages 1956-1957, 1997.

[7] J.M. Cruz-Hernández and V. Hayward. An approach to reduction of hysteresis in smart materials. In 1998 IEEE International Conference on Robotics and Automation, pages 1510$1515,1998$.

[8] C.A. Desoer and M. Vidyasagar. Feedback Systems: Input-Output Properties. Academic Press, 1975 .

[9] S. Fatikow and U. Rembold. Microsystem Technology and Microrobotics. Berlin ; New York : Springer, 1997.

[10] R.B. Gorbet and K.A. Morris. General dissipation in hysteretic systems. In Proceedings of the 37th IEEE Conference on Decision and Control, volume 3, pages 4133-4138, 1998.

[11] R.B. Gorbet, K.A. Morris, and D.W.L. Wang. Stability of control for the Preisach hysteresis model. In Proceedings of the 1997 IEEE International Conference on Robotics and Automation, volume 1, pages 241-247, 1997.

[12] D.J. Hill and P.J. Moylan. Stability results for nonlinear feedback systems. Automatica, 13:377-382, 1977. 
[13] D.J. Hill and P.J. Moylan. Dissipative dynamical systems: Basic input-output and state properties. Journal of the Franklin Institute, 309(5):327-357, May 1980.

[14] I.D. Mayergoyz. Mathematical Models of Hysteresis. Springer-Verlag, 1991.

[15] J.-F. Mougenet and V. Hayward. Limit cycle characterization, existence and quenching in the control of high performance hydraulic actuator. In Proc. IEEE International Conference on Robotics and Automation, volume 3, pages 2218-2223, 1995.

[16] S. O. Reza Moheimani and G. C. Goodwin. special issue on dynamics and control of smart structures. IEEE T. On Control Systems Technology, 9(1), 2001.

[17] A. Sebastian and S. Salapaka. $\mathrm{H}^{\infty}$ loop shaping design for nano-positioning. In American Control Conference, volume 5, pages 3708-3713, 2003.

[18] J.J. Slotine and W. Li. Applied Nonlinear Control. Prentice Hall, 1991. 